\title{
tRNA slippage at the tmRNA resume codon
}

\author{
MICHAEL J. TRIMBLE, AMY MINNICUS, and KELLY P. WILLIAMS \\ Department of Biology, Indiana University, Bloomington, Indiana 47405, USA
}

\begin{abstract}
The bacterial ribosome does not initiate translation on the mRNA portion of tmRNA; instead translation that had begun on a separate mRNA molecule resumes at a particular triplet on tmRNA (the resume codon). For at least two tRNAs that could pair with both the resume and -2 triplets on mutant tmRNAs, UAA (stop) as the second codon induced high-frequency -2 slippage on the resume codon in the $P$ site. The frameshift product was not detected when the -2 base was altered. Deficiency for ribosomal L9 protein, which affects other cases of frameshifting, had no significant effect. A special feature of this frameshifting is its dependence on a particular context, that of the tmRNA resume codon; it failed on the same sequence in a regular mRNA, and, more strikingly, at the second tmRNA codon. This focuses attention on the peculiar features expected of the slippage-prone state, such as unusual E-site filling, that might make the P-site resume codon:anticodon interaction especially unstable.
\end{abstract}

Keywords: tmRNA; ribosome; frameshift; E site; translation

\section{INTRODUCTION}

Frameshift errors during translation are rare (Kurland 1992). Recent structural data improve our understanding of the fidelity of frame maintenance; they reveal a multiplicity of contacts made by the small subunit of the ribosome to mRNA and A- and P-site tRNAs, including all 6 nucleotides of the adjacent codon:anticodon pairings, with additional clamping on both anticodon stems (Ogle et al. 2001; Yusupov et al. 2001; Stahl et al. 2002). The 3' mRNA flank enters through a clamping tunnel in the neck of the small subunit, and the 5' flank exits through a tunnel in the other side of the neck (Frank and Agrawal 2000; Yusupova et al. 2001). A different viewpoint on fidelity comes from its subversion; in special cases, through diverse mechanisms, translational frameshifting can reach efficiencies as high as 50\% (Farabaugh 1996; Baranov et al. 2002a). One vulnerable stage is when the A site is empty and P-site tRNA can slip on mRNA (Hansen et al. 2003); such cases are often promoted by a stop codon in the A site, which is generally decoded by a release factor more slowly than is a sense codon by a tRNA. Here we describe a special case of highfrequency frameshifting, P-site tRNA slippage on tmRNA, that provides new insight into frame maintenance.

tmRNA is a bacterial RNA with both tRNA-like and mRNA-like properties. Its ends form a tRNA-like structure

Reprint requests to: Kelly P. Williams, 1001 E. Third St., Bloomington, IN 47405, USA; e-mail: kellwill@indiana.edu; fax: (812) 855-6705.

Article and publication are at http://www.rnajournal.org/cgi/doi/ 10.1261/rna.7010904. sufficiently similar to tRNA ${ }^{\text {Ala }}$ to allow charging with alanine. It also contains a reading frame that is translated, but in a highly unusual manner. tmRNA ameliorates problems associated with ribosomes that have stalled during translation, for example at the end of an mRNA with no in-frame stop codon (nonstop mRNA; Keiler et al. 1996). The ribosome runs to the last intact codon of the nonstop mRNA (Williams et al. 1999), then switches to translation of the mRNA portion of tmRNA, the first codon of which is termed the resume codon (Fig. 1). The result of tmRNA translation is release of the stalled ribosome at a tmRNA stop codon and tagging of the protein product with a peptide that signals proteolysis of the entire tagged protein. Several events of tmRNA translation have no precedent in the translation of normal mRNAs: (1) an elongation cycle (with peptidyl transfer to the alanine at the $3^{\prime}$ end of tmRNA itself) occurs without A-site codon:anticodon pairing; (2) the ribosome is passed from an mRNA molecule to tmRNA, with the two RNAs sharing the coding for a single protein product; (3) the resume codon is selected by a process that differs in many ways from initiation at a start codon. Further study of these unusual phenomena, and the new one described here, can be expected to lead to a deeper understanding of normal ribosomal function.

An earlier study showed that tmRNA can function effectively with various triplets in the resume codon position, which are read according to the genetic code, but a mutant with a stop codon in that position is not used effectively (Williams et al. 1999). As a control for the latter result, a stop codon was placed in the second position, immediately 


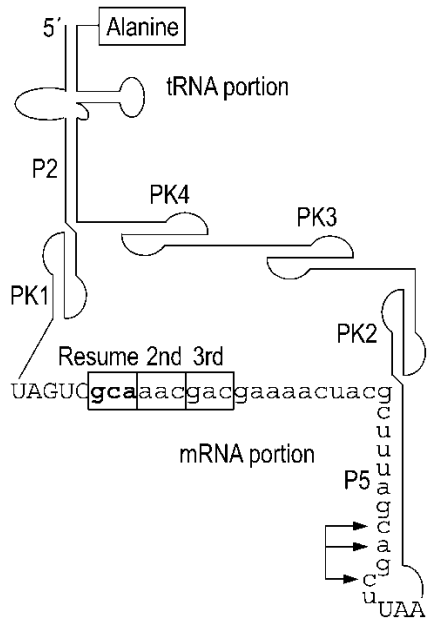

FIGURE 1. Features of Escherichia coli tmRNA. The coding portion is in lower case letters. In experiments here, the second and third codons were converted to stop codons. (P2, P5) base-paired stems; (PK1-4) pseudoknots; (arrows) a set of positions changed to prevent proteolysis of products translated in the standard reading frame (see Fig. 2).

following the resume codon. This mutant produced both the expected short tag and a second product whose mass was compatible with -2 slippage of the tRNA reading the resume codon. Here we provide experimental support for this idea, showing that the second product is not detected when the -2 base is changed, and showing that at least two tRNA species are subject to such slippage. Moreover we show that this slippage is specific to the resume codon position; strikingly, it fails at the next position, when the frameshifting sequence is moved $3 \mathrm{nt}$ in the $3^{\prime}$ direction. This suggests that the small subunit has an unusually loose hold on tmRNA when the resume codon is in the P site.

\section{RESULTS}

\section{Slippage of tRNA ${ }_{\text {VGA }}^{\text {Ser }}$ at the resume codon}

In an informative in vivo assay for tmRNA activity, an mRNA is induced in Escherichia coli in nonstop form (lacking an in-frame stop codon), by placing a strong transcriptional terminator within the reading frame (Keiler et al. 1996). The encoded reporter protein is subject to tmRNAdirected tagging, which increases its mass by more than $1000 \mathrm{D}$. The tag is engineered, by mutation of tmRNA far from the resume codon (Fig. 1), to prevent its normal proteolysis-inducing function and thereby allow positive detection of the tagged protein. Total reporter protein, which contains a six-histidine block, is purified on nickel resin for analysis by mass spectrometry. In such analysis for a tmRNA variant whose resume codon (UCA) specifies the $E$. coli tRNA ${ }_{\text {VGA }}^{\text {Ser }}(\mathrm{V}=$ uridine 5-oxyacetic acid; Fig. 2, mutant 2), the two main peaks are from untagged (oval) and tagged (triangle) forms of the reporter protein that resulted from translation to the last codon present in the majority of nonstop transcripts (Fig. 3, mutant 2; Williams et al. 1999). Related minor peaks (small circles) result from less frequent translation of the nonstop mRNA to one or two fewer codons or to one extra codon; these minor species can be untagged or tagged.

In another tmRNA mutant, the same UCA resume codon was followed by the UAA stop codon rather than a sense codon (Fig. 3, mutant 3). The triangle marks the expected tagging product from use of the zero frame that would add only two amino acids, the alanine transferred directly from tmRNA and the serine encoded by the resume codon. The exclamation point marks an unexpected product, representing approximately $30 \%$ of the tagging events, whose mass matches that expected from translation of the -2 reading frame of tmRNA. This product could arise by initial selection of the -2 triplet at $30 \%$ frequency or by frequent tRNA slippage from the standard resume triplet to the -2 triplet. The initial-selection hypothesis should not depend on the identity of the codon following the resume codon, but other cases of slippage of P-site tRNA are strongly dependent on the identity of following codon, and are enhanced when a stop codon is positioned in the A site (Hansen et al. 2003). Observing the -2 translation product when the second tmRNA codon is a stop codon, but not when it is a sense codon favors the hypothesis that the standard resume codon is selected uniformly, but the selected tRNA frequently undergoes a -2 slip in the $\mathrm{P}$ site when a stop codon is present in the $\mathrm{A}$ site. This would require tRNA ${ }_{\mathrm{VGA}}^{\mathrm{Ser}}$ to slip from the cognate UCA resume codon to the UCU triplet at the -2 position in apparent violation of wobble rules; however tRNA ${ }_{\text {VGA }}^{\text {Ser }}$ is known to efficiently recognize the UCU triplet during in vitro translation (Yamada et al. 2003).

The -2 slippage hypothesis was further tested by altering the base at the -2 position. Among natural tmRNA resume region sequences, this position is the least conserved (Williams et al. 1999), suggesting that its alteration might not impair tmRNA utilization. As expected, changing the -2 position from $\mathrm{U}$ to $\mathrm{A}$ (without the early stop codon) did not inhibit tmRNA action (Fig. 3, mutant 4). Combining this -2 alteration with a stop codon following the resume codon, the shift product was not observed (Fig. 3, mutant 5 ), in support of the tRNA slippage hypothesis.

\section{Shift fails on the same sequence at the next tmRNA triplet}

Other codons in tmRNA close to the resume codon may be susceptible to tRNA slippage. To test for slippage at the second codon of tmRNA, an 11-nt sequence from the resume region of the frameshifting tmRNA (mutant 3) was moved $3 \mathrm{nt}$ in the $3^{\prime}$ direction; no shifting was detected (Fig. 2, mutant 7). The strength of the result is somewhat diminished in this case by the lower level of tmRNA utilization, which raises the detection limit; frameshifting at a frequency of $5 \%$ or lower would have escaped detection. 
A

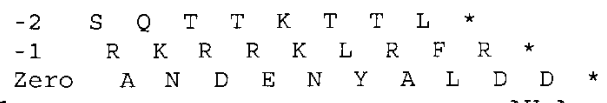

1 uagucgcaaacgacgaaaacuacgcuuuagAJgAuuaa

$\mathrm{B}$

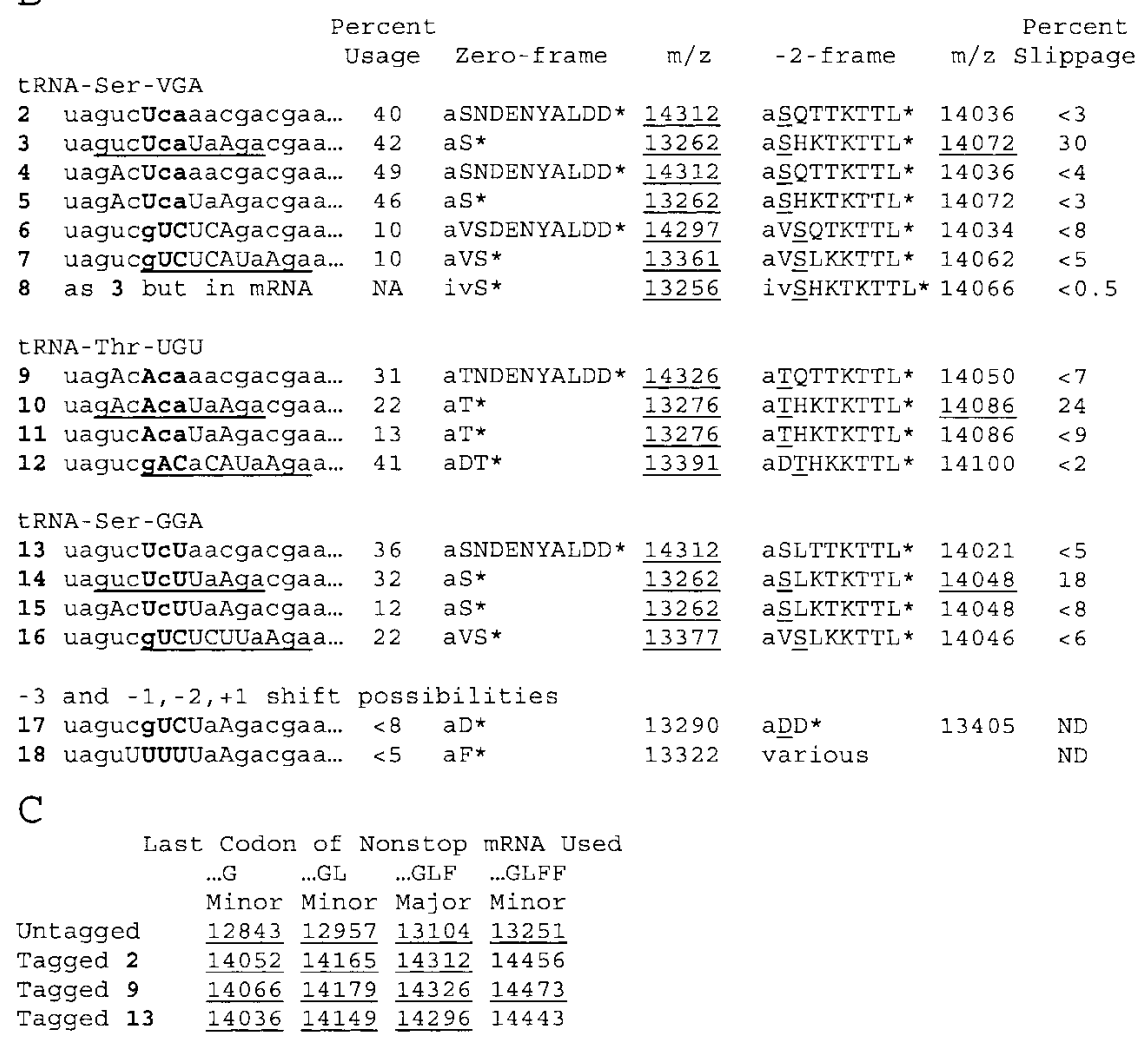

FIGURE 2. Frameshifting assay of mutant tmRNAs. (A) Coding by three reading frames. (Upper case) mutation relative to wild-type; (bold) mutant number and resume triplet. (B) Sets of mutant tmRNAs testing -2 frameshifting for three different resume codons, with expected masses for tagging products. (Underline) 11-nt block shifted by $3 \mathrm{nt}$. Sequence of expected tag (the first tag residue, alanine transferred from tmRNA itself, is in lower case) and calculated mass of protonated form of tagged reporter for 0 and -2 frames, with observed products (all within $3 \mathrm{D}$ of expected mass) underlined. See Materials and Methods for determination of usage and slippage. $(C)$ Masses expected for protonated states of major untagged and (zeroframe) tagged species and minor species resulting from translation of nonstop mRNA ending at different codons, with observed products (within $3 \mathrm{D}$ of expected mass) underlined.

\section{Shift fails on the same sequence in an mRNA $\bullet$ tmRNA fusion}

A 39-nt segment of mutant 3, starting at 6 nt upstream of the resume codon, was fused to the reporter mRNA in frame with the resume codon, so that the frameshifting sequence could be tested in the context of a regular mRNA (mutant 8). No frameshifting was detected, with an exceptionally low detection limit $(<0.5 \%$; Fig. 4$)$.

\section{Slippage with two other resume codon sequences}

Slippage might be due to peculiar features of tRNA ${ }_{\text {VGA }}^{\text {Ser }}$, such as its long variable loop. A set of mutants was designed to test whether tRNA ${ }_{\text {UGU }}^{\mathrm{Thr}}$, with the more usual variable loop size of $5 \mathrm{nt}$, would behave like tRNA ${ }_{\text {VGA. }}^{\text {Ser }}$ The ACA resume codon reduced the utilization of tmRNA (Fig. 5 , mutant 9), but frameshifting was still detectable when a stop codon was placed at the second position (Fig. 5, mutant 10). The signal was reproducible (inset), accounting for approximately $24 \%$ of tagging events. Changing the -2 base prevented the formation of this product (Fig. 5, mutant 11). Thus tRNA $^{\text {Thr }}{ }_{\text {UGU }}$ like tRNA ${ }_{\text {VGA }}^{\text {Ser }}$ is subject to -2 slippage at the resume codon.

Slippage of tRNA ${ }_{\text {UGU }}^{\text {Thr }}$ was not detected at the second tmRNA codon, when an 11-nt segment from the frameshifting sequence was moved $3 \mathrm{nt}$ in the 3 ' direction (Fig. 5, mutant 12). This case argues, as above for tRNA ${ }_{\mathrm{VGA}}^{\mathrm{Ser}}$, that only the resume codon is susceptible to high-level shifting. The result is stronger in this case, because this tmRNA was more efficiently used, reducing the detection limit to $2 \%$.

A similar set of mutants yielded essentially the same results with UCU at the resume codon (Fig. 2, mutants 13-16), which would suggest that tRNA ${ }_{\text {GGA }}^{\mathrm{Ser}}$ is also subject to slippage, but this conclusion is not firm because the same tRNA species examined above ( $\mathrm{tRNA}_{\mathrm{VGA}}^{\mathrm{Ser}}$ ) is known to recognize the UCU codon and may have been responsible for this case of slippage too.

\section{Examining the effect of ribosomal protein $\mathrm{L} 9$}

Deletion of the gene ( $r p l I)$ for the ribosomal protein L9 has been found to produce two- to threefold increases in -1 frameshifting and improve frameshifting in other systems (Herr et al. 2000, 2001). All of our tmRNA mutants 1-16 were tested in a $\Delta r p l I$ background, but no significant differences relative to the isogenic $r p l I^{+}$strain were observed for -2 tRNA slippage. Two examples are shown in Figure 6; in the $\Delta$ rplI strain, slippage was $33 \%$ for mutant 3 and undetected for mutant 12 .

\section{Avoidance of slippery sequences in natural tmRNAs}

The phenomenon described here is a new case of tRNA slippage, but does it have relevance to normal tmRNA translation? On the one hand, detection of frameshifting here required the artificial introduction of a shift-prone resume codon sequence followed by an early stop codon, 


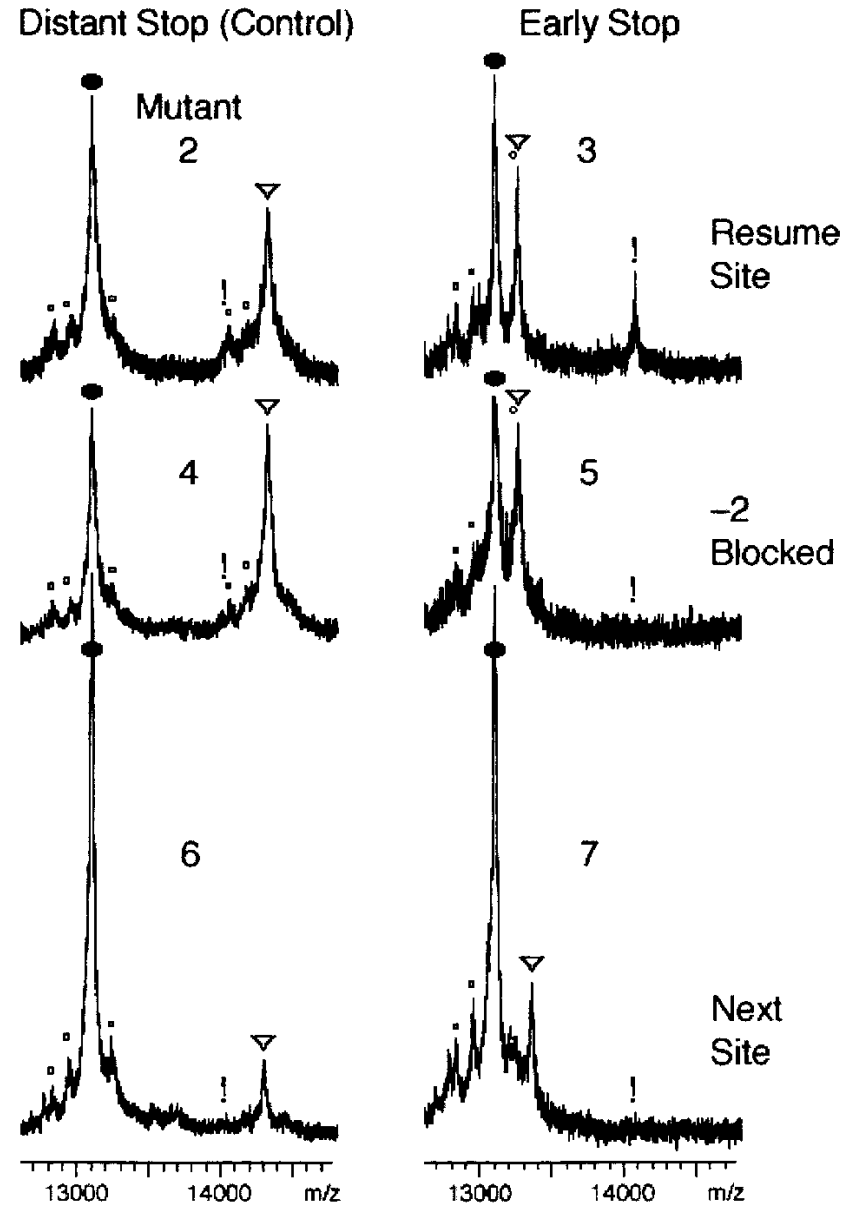

FIGURE 3. Slippage of tRNA ${ }_{\text {VGA }}^{\mathrm{Ser}}$ at resume codon. MALDI-TOF traces of reporter protein with various tmRNA mutants in strain X90 $s s r A:$ cat. Symbols mark expected positions of reporter protein species: (oval) major untagged; (small circles) minor species resulting from last use of other codons of nonstop mRNA; (triangle) major 0 -frame tagging product; (exclamation mark) major -2-frame tagging product. Use of the -2 frame was observed only when -2 slippage was possible at the resume codon followed by a stop codon; the mass for the peak near the exclamation mark for mutant 2 matched that for a minor 0 -frame tagged species and not the -2 -frame product. See Figure 2 for a description of the mutants.

and therefore might not be significant during translation of natural tmRNAs. On the other hand, our assay is not highly sensitive; low levels of slippage may occur even without an early stop codon and interfere with tmRNA activity. It can be noted that natural tmRNAs appear to avoid shift-prone sequences at the resume codon. In the -2 to +3 region of natural tmRNA resume codons, as called at the tmRNA Web site (Gueneau de Novoa and Williams 2004), G is always present at the +1 position, but any base is tolerated at the other positions. Insisting on the $G$ at +1 , and with simple wobble rules, the following patterns can be deduced for $-2,-1$, and +1 shift-prone sequences: GNGNR (where $\mathrm{N}$ is the same base at both positions), GGGR and GGGR (resume codons underlined). With equal probability for bases at ambiguous positions, each of these patterns would be expected once in 32 random sequences of the same size. Instead, none of these patterns are found at the resume codon for any of the 380 natural tmRNA sequences available at the tmRNA Web site.

\section{DISCUSSION}

\section{tRNA slippage by -2 on the resume codon in the $P$ site}

A possible explanation for use of the -2 frame, not involving slippage, would be that the first tRNA to read tmRNA pairs initially with the -2 triplet upon entry into the A site. On a normal mRNA, such a model would be implausible because two thirds of the -2 triplet would be occluded by P-site tRNA, but this objection does not hold for the resume codon of tmRNA in the A site. Nonetheless this nonslippage proposal is untenable, because the -2 triplet was not used when the same sequence as in a frameshifting mutant was available for initial pairing (cf. mutants 2 and 3 or 9 and 10).

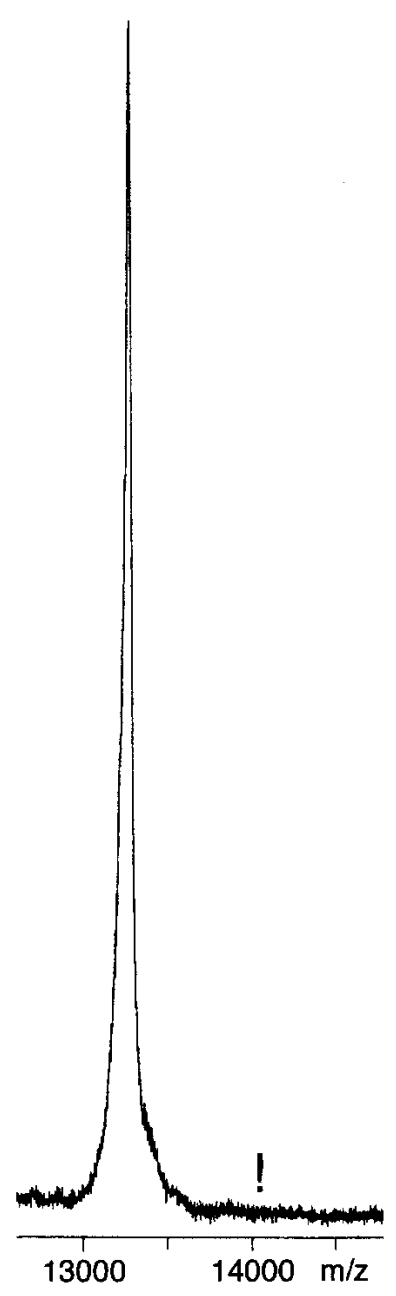

FIGURE 4. Failure of tRNA ${ }_{\text {vGA }}^{\text {Ser }}$ slippage in an mRNA/tmRNA fusion. Analysis of mutant 8 is as in Figure 3. 


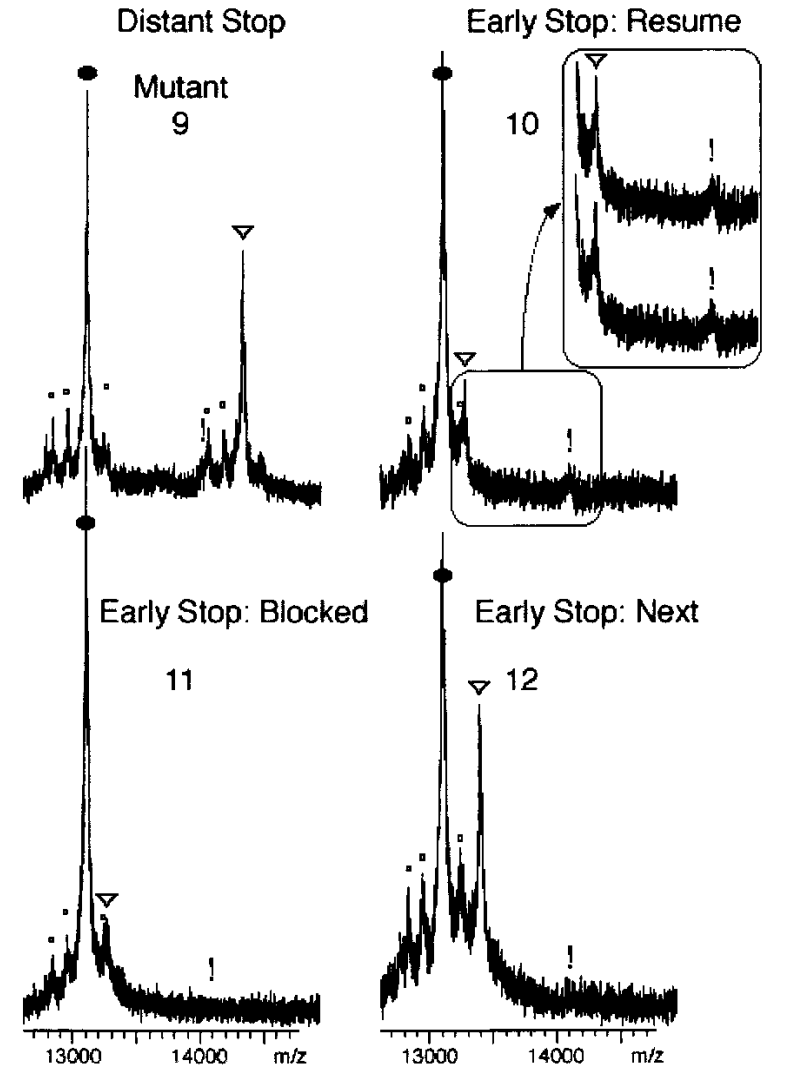

FIGURE 5. Slippage of tRNA ${ }_{\text {UGU }}^{\text {Thr }}$ at resume codon. Explanation are as in Figure 3. Inset shows 0 - and -2-frame tagging products from duplicate independent assays.

One summary of our results is that the -2 triplet was used only when the same tRNA could read both it and the resume triplet, which argues that tRNAs pair first with the resume triplet as usual, then slip back frequently. Whether this slippage occurs when the resume codon is in the A site or the $\mathrm{P}$ site is addressed by its occurrence with a stop codon in the second position, but not with a sense codon there. The second codon should only be effective when it is being decoded in the A site and the resume codon therefore already translocated to the $\mathrm{P}$ site. Stop codons in the A site have been found to improve several cases of P-site slippage relative to sense codons, presumably because they are more slowly decoded, leaving more time for the event. In one study of -2 frameshifting (Weiss et al. 1990), the UGA stop codon was found to be more effective than UAA, suggesting that it may be possible to boost the already frequent frameshifting observed here.

\section{Unusual features of the shift-prone state}

The most striking aspect of the frameshifting studied here is its context dependence. This point is best made by the occurrence of frameshifting when the resume codon is in the $\mathrm{P}$ site and its failure when the next tmRNA codon is there.
Two general hypotheses, not mutually exclusive, can be advanced to explain this position specificity: (1) codon 2 is decoded as a stop codon much more slowly than is codon 3 , providing an exceptionally long period for otherwise rare tRNA slippage, and (2) the P-site codon:anticodon pairing is much less stable for the resume codon than for the next codon.

Several features of the frameshifting state are notable (Fig. 7). In solution, the reading frame is flanked by a pseudoknot apparently coaxially stacked with a double helix, both upstream (P2-PK1) and at its $3^{\prime}$ end (P5-PK2; Fig. 1; Williams and Bartel 1996; Felden et al. 1997). These stempseudoknots were modeled into a low-resolution image of a very early stage of tmRNA loading, when the mRNA portion was on the outer surface of the small subunit (Valle et al. 2003), but there is no information about their status during translation of tmRNA.

Downstream pseudoknots can be potent frameshifting signals. However, in tmRNA, the downstream stem-pseudoknot structure P5-PK2 begins at the +19 position of the reading frame, so distant that any effect on frameshifting would be more likely at the second codon than at the resume codon, opposite to our observation. A -1 frameshiftpromoting pseudoknot from coronavirus is active in E. coli at the +13 position but much less so at +16 , and a -1 frameshift-promoting stem-loop of E. coli dnaX has optimal activity at +12 , decreasing steadily with longer spacers (Brierley et al. 1997; Larsen et al. 1997).

The upstream P2-PK1 unit is also rather distant, with an 11-nt spacer from the resume codon in E. coli. Nonetheless, if it remains intact during tmRNA translation, it might be responsible for a looser hold on tmRNA; as an example, it could prevent the upstream mRNA region from following the usual track, an effect that might be transmitted back to the $\mathrm{P}$ site. Difficulty filling the mRNA track could be exac-
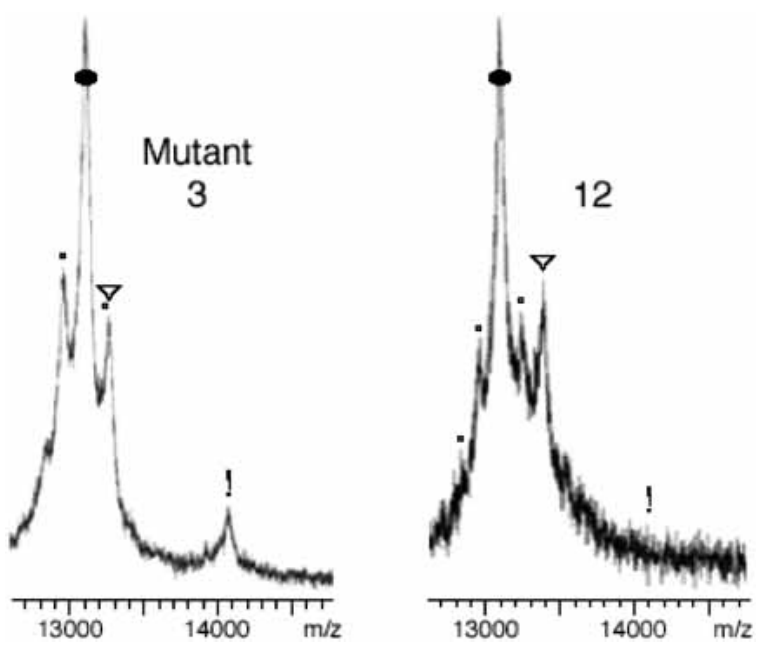

FIGURE 6. Slippage unchanged with ribosomes lacking L9 protein. Analysis of two tmRNA mutants in strain KW1110 ( $\Delta$ rplI) is as in Figure 3. 


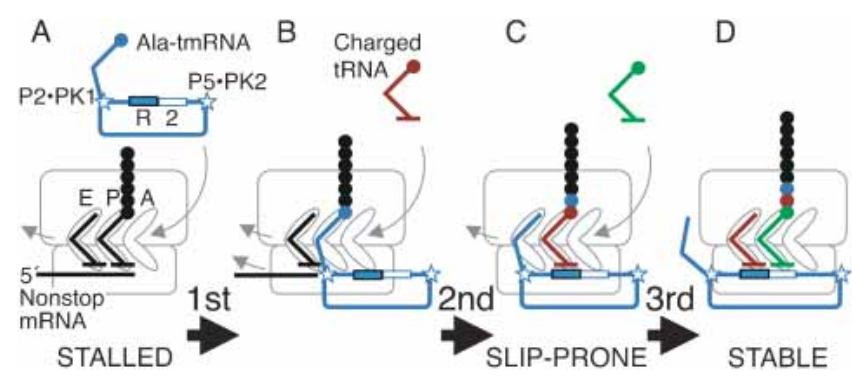

FIGURE 7. Model for first three elongation cycles on tmRNA. $(A)$ When the ribosome stalls at the end of nonstop mRNA, alanylated tmRNA enters (boxes mark its resume $[R]$ and second codons; stars mark flanking structures observed in solution). (B) After one elongation cycle, the resume codon is presented normally in the A site, but the small-subunit P site is filled abnormally. $(C)$ After a second elongation cycle, the nonstop mRNA and its last-specified tRNA have been ejected, the small-subunit $\mathrm{E}$ site is filled abnormally, and the P-site tRNA is prone to slippage. $(D)$ After a third elongation cycle, the E site is filled properly with tRNA and the P-site codon:anticodon pairing is stable.

erbated by the topological constraint imposed by the looping of the mRNA domain. A cryo-EM image suggests that at least before loading into the ribosome is complete, the diameter of this loop is comparable to that of the neck of the small subunit (Valle et al. 2003). However our data insist that any shift-promoting features acting when the resume codon is in the P site are corrected after a single elongation cycle places the next codon there. One feature expected to change so suddenly is E-site filling.

\section{Correlation of P-site slippage with unusual E-site filling}

In normal translation, tRNA in the P site is moved to the exit (E) site after one elongation cycle and is ejected after one more cycle. In the crystal structure of the loaded ribosome, seven contacts were noted for E-site tRNA with the large subunit and five with the small subunit, mostly at the anticodon loop (Yusupov et al. 2001). These position the E-site tRNA alongside the P-site tRNA, approaching most closely in the small subunit. The two tRNAs are not in direct contact but are linked by the $16 \mathrm{~S}$ rRNA helices H24, $\mathrm{H} 28$, and $\mathrm{H} 29$ that they both contact directly through their anticodon loops. Another avenue of $\mathrm{E}$ - and P-site linkage is through the mRNA. A single possible contact was noted between the mRNA and E-site tRNA in the crystal structure, but this was a noncognate tRNA. Even this noncognate E-site anticodon was close enough to the codon that the cognate interaction is structurally plausible, and there is biochemical evidence for codon:anticodon specificity in the E site (Rheinberger et al. 1986; Lill and Wintermeyer 1987). E-site tRNA is thus sufficiently connected to the P site that a role in promoting the stability of P-site codon:anticodon pairing is a very real possibility. This role has been proposed before, from a case where frameshifting is facilitated by an internal Shine-Dalgarno interaction that extends into the E-site codon and should affect E-site tRNA, perhaps repositioning or even prematurely ejecting it (Blaha and Nierhaus 2001; Baranov et al. 2002b). We make the same proposal based on the unusual E-site filling expected for tmRNA during the slippage-prone state.

It is not known precisely when nonstop mRNA and its last-specified tRNA are ejected from the ribosome, but it would be reasonable to expect them both to be absent after two elongation cycles have placed the resume codon in the $\mathrm{P}$ site (Fig. 7). If so, there would be no true E-site tRNA present at the time of slippage, and the deacylated tRNAlike domain of tmRNA itself would be the closest substitute. tmRNA contains a canonical acceptor stem and T stemloop that could make all the contacts that E-site tRNA makes with the large subunit. However tmRNA contains no anticodon loop (an internal bulge in the E. coli tmRNA that has been likened by some to an anticodon loop is not conserved evolutionarily). Furthermore, the equivalent to an anticodon stem (P2) makes a much larger angle with the acceptor arm $\left(\sim 120^{\circ}\right)$ than the corresponding angle in tRNA $\left(\sim 90^{\circ}\right)$, in the presence or absence of its cofactor SmpB (Stagg et al. 2001; Gutmann et al. 2003). This striking shape difference suggests that tmRNA would be incapable of properly filling the small-subunit E site. If E-site tRNA normally helps prevent tRNA:mRNA slippage in the P site, this could explain the susceptibility of the resume codon to slippage. After an additional elongation cycle, when the second tmRNA codon is in the P site and slippage is no longer frequent, the $\mathrm{E}$ site should be filled normally with deacylated tRNA.

\section{Attempts at other shift distances}

Frameshifting by -2 slippage of P-site tRNA on regular mRNA has been observed previously, as well as forward hops from +5 and +6 to as large as +50 , but examples of -1 and +1 frameshifts are much more common (Weiss et al. 1990; Farabaugh 1996). To investigate other possible shift distances at the resume codon, two mutants were constructed; for one, a -3 shift might have been possible, and for the other, $-2,-1$, or +1 shifts might have occurred (Fig. 2, mutants 17 and 18). Unfortunately, frameshifting could not be assessed in these mutants, because neither produced even the zero-frame tagging product detectably. The possibility of other shift distances at the resume codon thus remains untested. One attractive system to test in the future is -1 slippage on GGGA, which has been well studied in the context of a regular mRNA (Herr et al. 2001).

\section{Ribosomal protein L9}

Deletion of the gene for the ribosomal protein L9 has been found to produce two- to threefold increases in -1 frameshifting, larger increases in forward hopping events of +5 or 
more, and suppression of weakened versions of the 50-nt translational bypass on phage T4 gene 60 mRNA (Herr et al. $2000,2001)$. L9 has a highly elongated shape in solution and in a ribosome crystal structure it protrudes perpendicularly from the surface of the large subunit near the mRNA exit channel (Hoffman et al. 1996; Yusupov et al. 2001). It is not known how a protein in this position affects mRNA:tRNA pairing in the small subunit. It may influence the nearby $\mathrm{E}$ site. It has also been proposed that L9 fends off the trailing ribosome in a polysome, and that closer approach of this neighbor ribosome in the absence of L9 destabilizes mRNA: tRNA pairing (Herr et al. 2001). If this latter proposal is true, the failure of L9 deficiency to affect slippage on the resume codon can be easily explained by prior release of the tmRNA monosome from the nonstop mRNA polysome (Fig. 7).

\section{Conclusions}

The frameshifting observed here was artificial in that a slippery resume codon and a stop codon at the second position were required for it to become frequent. Frameshifting cannot therefore be considered an important aspect of normal tmRNA function, although we do note that natural tmRNA sequences avoid slippery resume codons. The value of this observation is the insight it provides into both the normal ribosomal function of frame maintenance and the interesting early stages of tmRNA usage, for which many mechanistic and structural details are lacking. It is at least clear that the first elongation cycle on tmRNA is highly unusual; peptidyl transfer occurs without proper A-site codon:anticodon pairing and the mRNA portion is freshly loaded into the small subunit, placing the resume codon into the A site. Previous studies had indicated that the second elongation cycle might be fairly normal, in that several different tRNAs could decode various resume codon triplets in the A site. Instead, our results show that translation has not returned to normal even after the second cycle is complete; tRNA is prone to high-frequency slippage on the resume codon in the $\mathrm{P}$ site. However a single additional elongation cycle suffices to restore normalcy, at least as regards slippage.

Other cases of tRNA slippage combine contributing factors, such as slippage-prone tRNAs or mRNA sequences, downstream mRNA structures, frame-internal Shine-Dalgarno pairings, and slow A-site filling, that could be assembled at any position in an mRNA to promote slippage. A unique aspect of the present case is its context specificity, its dependence on the placement of the tmRNA resume codon in the $\mathrm{P}$ site. tmRNA presents several unusual features that might be invoked in an explanation of slippage, but E-site filling is particularly attractive. The $\mathrm{E}$ site is close by and physically linked to the P-site codon:anticodon pairing. tmRNA can be expected to fill it improperly during the slippage-prone state, yet this should be corrected by normal filling with regular tRNA in the stable state after the next elongation cycle (Fig. 7). The hypothesis that tmRNA fills the E site abnormally provides yet another motive, among many, for structural analysis of tmRNA translation.

\section{MATERIALS AND METHODS}

\section{Strains}

E. coli X90 ssrA::cat (Keiler et al. 1996) was transduced to kanamycin resistance by P1 phage grown on AH156 ( $\Delta r p l I:: k a n$; Herr et al. 2000) and loss of $r p l I$ was confirmed in the resulting strain KW1110 by PCR of genomic DNA.

\section{Plasmids}

The vector p199T was constructed from pKW8 as described (Williams et al. 1999), producing a tmRNA gene in which $20 \mathrm{bp}$ in the resume region were replaced with a segment flanked by BsaI sites. p199T was digested with BsaI and complementary pairs of 24-nt oligonucleotides were inserted to produce desired changes, all confirmed by subsequent sequencing. Resulting low-copy plasmids encode the mutant tmRNA (also containing the mutations changing the last two tag codons from Ala-Ala to Asp-Asp to prevent proteolysis of tagged protein) under control of the native promoter and terminator. For mutant 8 (Figs. 2, 4), 39 bp containing the whole reading frame from the frameshifting tmRNA mutant 3 and $6 \mathrm{bp}$ of upstream sequence replaced the final codon of the nonstop coding region of the reporter plasmid pPW500 (Keiler et al. 1996), whose terminator stem-loop was inactivated while retaining the original coding.

\section{In vivo frameshift assay}

E. coli X90 ssrA::cat or KW1110 was doubly transformed with a low-copy tmRNA-encoding plasmid and the high-copy plasmid pPW500 encoding 6-His-containing reporter protein with a nonstop mRNA. Reporter protein was induced and purified on nickel resin as described (Williams et al. 1999). Samples were mixed with myoglobin and analyzed by MALDI-TOF mass spectrometry, using myoglobin and the major untagged reporter species as internal standards for mass calibration. The major untagged reporter species, and the related species tagged in frame 0 or in frame -2 , were quantitated as the area under the peak, or when undetected, by the estimated minimum detectable peak area as an upper bound. tmRNA usage was evaluated as the percentage of the tagged species to the total for all three species, and slippage as the percentage of -2-frame tagging product to the total for the tagged species. Although mass spectrometry can be nonquantitative when comparing unrelated molecules, the protein species analyzed here are very closely related; they all contain the same 117-amino-acid C-terminal sequence, which comprises no less than $91 \%$ of the mass of any of the species analyzed.

\section{ACKNOWLEDGMENTS}

We thank John Atkins (University of Utah) for suggesting the test of L9 and providing the strain AH156. This work was supported by an NIH grant. 
The publication costs of this article were defrayed in part by payment of page charges. This article must therefore be hereby marked "advertisement" in accordance with 18 USC section 1734 solely to indicate this fact.

Received January 2, 2004; accepted February 4, 2004.

\section{REFERENCES}

Baranov, P.V., Gesteland, R.F., and Atkins, J.F. 2002a. Recoding: Translational bifurcations in gene expression. Gene 286: 187-201. - 2002b. Release factor 2 frameshifting sites in different bacteria. EMBO Rep. 3: 373-377.

Blaha, G. and Nierhaus, K.H. 2001. Features and functions of the ribosomal E site. Cold Spring Harbor Symp. Quant. Biol. 66: 135146.

Brierley, I., Meredith, M.R., Bloys, A.J., and Hagervall, T.G. 1997. Expression of a coronavirus ribosomal frameshift signal in Escherichia coli: Influence of tRNA anticodon modification on frameshifting. J. Mol. Biol. 270: 360-373.

Farabaugh, P.J. 1996. Programmed translational frameshifting. Annu. Rev. Genet. 30: 507-528.

Felden, B., Himeno, H., Muto, A., McCutcheon, J.P., Atkins, J.F., and Gesteland, R.F. 1997. Probing the structure of the Escherichia coli 10Sa RNA (tmRNA). RNA 3: 89-103.

Frank, J. and Agrawal, R.K. 2000. A ratchet-like inter-subunit reorganization of the ribosome during translocation. Nature 406: 318 322.

Gueneau de Novoa, P. and Williams, K.P. 2004. The tmRNA website: Reductive evolution of tmRNA in plastids and other endosymbionts. Nucleic Acids Res. 32: D104-D108.

Gutmann, S., Haebel, P.W., Metzinger, L., Sutter, M., Felden, B., and Ban, N. 2003. Crystal structure of the transfer-RNA domain of transfer-messenger RNA in complex with SmpB. Nature 424: 699703.

Hansen, T.M., Baranov, P.V., Ivanov, I.P., Gesteland, R.F., and Atkins, J.F. 2003. Maintenance of the correct open reading frame by the ribosome. EMBO Rep. 4: 499-504.

Herr, A.J., Gesteland, R.F., and Atkins, J.F. 2000. One protein from two open reading frames: Mechanism of a $50 \mathrm{nt}$ translational bypass. $E M B O ~ J .19: 2671-2680$.

Herr, A.J., Nelson, C.C., Wills, N.M., Gesteland, R.F., and Atkins, J.F. 2001. Analysis of the roles of tRNA structure, ribosomal protein L9, and the bacteriophage T4 gene 60 bypassing signals during ribosome slippage on mRNA. J. Mol. Biol. 309: 1029-1048.

Hoffman, D.W., Cameron, C.S., Davies, C., White, S.W., and Ra- makrishnan, V. 1996. Ribosomal protein L9: A structure determination by the combined use of X-ray crystallography and NMR spectroscopy. J. Mol. Biol. 264: 1058-1071.

Keiler, K.C., Waller, P.R., and Sauer, R.T. 1996. Role of a peptide tagging system in degradation of proteins synthesized from damaged messenger RNA. Science 271: 990-993.

Kurland, C.G. 1992. Translational accuracy and the fitness of bacteria. Annu. Rev. Genet. 26: 29-50.

Larsen, B., Gesteland, R.F., and Atkins, J.F. 1997. Structural probing and mutagenic analysis of the stem-loop required for Escherichia coli dnaX ribosomal frameshifting: Programmed efficiency of 50\%. J. Mol. Biol. 271: 47-60.

Lill, R. and Wintermeyer, W. 1987. Destabilization of codon-anticodon interaction in the ribosomal exit site. J. Mol. Biol. 196: 137148.

Ogle, J.M., Brodersen, D.E., Clemons Jr., W.M., Tarry, M.J., Carter, A.P., and Ramakrishnan, V. 2001. Recognition of cognate transfer RNA by the 30S ribosomal subunit. Science 292: 897-902.

Rheinberger, H.J., Sternbach, H., and Nierhaus, K.H. 1986. Codonanticodon interaction at the ribosomal E site. J. Biol. Chem. 261: 9140-9143.

Stagg, S.M., Frazer-Abel, A.A., Hagerman, P.J., and Harvey, S.C. 2001. Structural studies of the tRNA domain of tmRNA. J. Mol. Biol. 309: 727-735.

Stahl, G., McCarty, G.P., and Farabaugh, P.J. 2002. Ribosome structure: Revisiting the connection between translational accuracy and unconventional decoding. Trends Biochem. Sci. 27: 178-183.

Valle, M., Gillet, R., Kaur, S., Henne, A., Ramakrishnan, V., and Frank, J. 2003. Visualizing tmRNA entry into a stalled ribosome. Science 300: $127-130$.

Weiss, R.B., Dunn, D.M., Atkins, J.F., and Gesteland, R.F. 1990. Ribosomal frameshifting from -2 to +50 nucleotides. Prog. Nucleic Acid Res. Mol. Biol. 39: 159-183.

Williams, K.P. and Bartel, D.P. 1996. Phylogenetic analysis of tmRNA secondary structure. RNA 2: 1306-1310.

Williams, K.P., Martindale, K.A., and Bartel, D.P. 1999. Resuming translation on tmRNA: A unique mode of determining a reading frame. EMBO J. 18: 5423-5433.

Yamada, Y., Matsugi, J., and Ishikura, H. 2003. tRNA(1)(Ser)(G(34)) with the anticodon GGA can recognize not only UCC and UCU codons but also UCA and UCG codons. Biochim. Biophys. Acta 1626: $75-82$.

Yusupov, M.M., Yusupova, G.Z., Baucom, A., Lieberman, K., Earnest, T.N., Cate, J.H., and Noller, H.F. 2001. Crystal structure of the ribosome at $5.5 \AA$ resolution. Science 292: 883-896.

Yusupova, G.Z., Yusupov, M.M., Cate, J.H., and Noller, H.F. 2001. The path of messenger RNA through the ribosome. Cell 106: 233 241. 

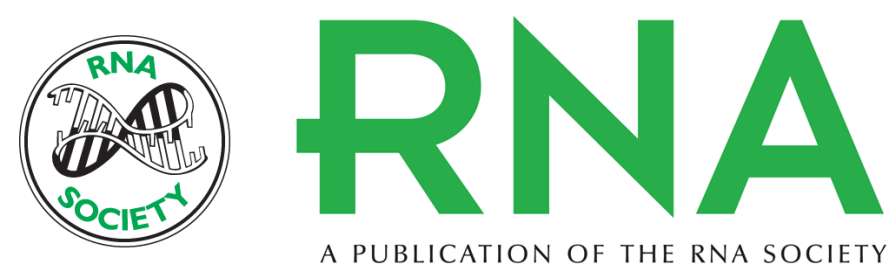

A PUBLICATION OF THE RNA SOCIETY

\title{
tRNA slippage at the tmRNA resume codon
}

\author{
MICHAEL J. TRIMBLE, AMY MINNICUS and KELLY P. WILLIAMS
}

RNA 2004 10: 805-812

References This article cites 28 articles, 9 of which can be accessed free at:

http://rnajournal.cshlp.org/content/10/5/805.full.html\#ref-list-1

\section{License} Email Alerting
Service 